\title{
THE DIMENSION OF SOME AFFINE DELIGNE-LUSZTIG VARIETIES
}

\author{
BY EVA VIEHMANN
}

\begin{abstract}
We prove Rapoport's dimension conjecture for affine Deligne-Lusztig varieties for $G L_{h}$ and superbasic $b$. From this case the general dimension formula for affine Deligne-Lusztig varieties for special maximal compact subgroups of split groups follows, as was shown in a recent paper by Görtz, Haines, Kottwitz, and Reuman.
\end{abstract}

(c) 2006 Elsevier Masson SAS

RÉSUMÉ. - On démontre la conjecture de Rapoport sur la dimension des variétés de Deligne-Lusztig affines pour $G L_{h}$ et $b$ superbasique. Ce cas implique la formule générale pour la dimension des variétés de Deligne-Lusztig affines pour des sous-groupes compacts maximaux de groupes déployés, résultat démontré dans un article récent de Görtz, Haines, Kottwitz et Reuman.

(c) 2006 Elsevier Masson SAS

\section{Introduction}

Let $k$ be a finite field with $q=p^{r}$ elements and let $\bar{k}$ be an algebraic closure. Let $F=k((t))$ and let $L=\bar{k}((t))$. Let $\mathcal{O}_{F}$ and $\mathcal{O}_{L}$ be the valuation rings. We denote by $\sigma: x \mapsto x^{q}$ the Frobenius of $\bar{k}$ over $k$ and also of $L$ over $F$.

Let $G$ be a split connected reductive group over $k$. Let $A$ be a split maximal torus of $G$ and $W$ the Weyl group of $A$ in $G$. For $\mu \in X_{*}(A)$ let $t^{\mu}$ be the image of $t \in \mathbb{G}_{m}(F)$ under the homomorphism $\mu: \mathbb{G}_{m} \rightarrow A$. Let $B$ be a Borel subgroup of $G$ containing $A$. We write $\mu_{\text {dom }}$ for the dominant element in the orbit of $\mu \in X_{*}(A)$ under the Weyl group of $A$ in $G$.

We recall the definitions of affine Deligne-Lusztig varieties from [6,1]. Let $K=G\left(\mathcal{O}_{L}\right)$ and let $X=G(L) / K$ be the affine Grassmannian. The Cartan decomposition shows that $G(L)$ is the disjoint union of the sets $K t^{\mu} K$ where $\mu \in X_{*}(A)$ is a dominant coweight. For an element $b \in G(L)$ and dominant $\mu \in X_{*}(A)$, the affine Deligne-Lusztig variety $X_{\mu}(b)$ is the locally closed reduced $\bar{k}$-subscheme of $X$ defined by

$$
X_{\mu}(b)(\bar{k})=\left\{g \in G(L) / K \mid g^{-1} b \sigma(g) \in K t^{\mu} K\right\} .
$$

Left multiplication by $g \in G(L)$ induces an isomorphism between $X_{\mu}(b)$ and $X_{\mu}\left(g b \sigma(g)^{-1}\right)$. Thus the isomorphism class of the affine Deligne-Lusztig variety only depends on the $\sigma$-conjugacy class of $b$.

There is an algebraic group over $F$ associated to $G$ and $b$ whose $R$-valued points (for any $F$-algebra $R$ ) are given by

$$
J(R)=\left\{g \in G\left(R \otimes_{F} L\right) \mid g^{-1} b \sigma(g)=b\right\} .
$$

ANNALES SCIENTIFIQUES DE L'ÉCOLE NORMALE SUPÉRIEURE 0012-9593/03/@ 2006 Elsevier Masson SAS. All rights reserved. 
There is a canonical $J(F)$-action on $X_{\mu}(b)$.

Let $\rho$ be the half-sum of the positive roots of $G$. By $\operatorname{rk}_{F}$ we denote the dimension of a maximal $F$-split subtorus. Let $\operatorname{def}_{G}(b)=\operatorname{rk}_{F} G-\operatorname{rk}_{F} J$. Let $\nu \in X_{*}(A)_{\mathbb{Q}}$ be the Newton point of $b$, compare [3]. For nonempty affine Deligne-Lusztig varieties the dimension is given by the following formula. Note that there is a simple criterion by Kottwitz and Rapoport (see [5]) to decide whether an affine Deligne-Lusztig variety is nonempty.

THEOREM 1.1. - Assume that $X_{\mu}(b)$ is nonempty. Then

$$
\operatorname{dim}\left(X_{\mu}(b)\right)=\langle\rho, \mu-\nu\rangle-\frac{1}{2} \operatorname{def}_{G}(b) .
$$

Rapoport conjectured this in [7], Conjecture 5.10 in a different form. For the reformulation compare [4]. In [9], Reuman verifies the formula for some small groups and $b=1$. For $G=G L_{n}$, minuscule $\mu$ and over $\mathbb{Q}_{p}$ rather than over a function field, the Deligne-Lusztig varieties have an interpretation as reduced subschemes of moduli spaces of $p$-divisible groups. In this case, the corresponding dimension formula is shown by de Jong and Oort (see [2]) if $b \sigma$ is superbasic and in [10] for general $b \sigma$. In [1] 2.15, Görtz, Haines, Kottwitz, and Reuman prove Theorem 1.1 for all $b \in A(L)$. They also show in 5.8 that if there is a Levi subgroup $M$ of $G$ such that $b \in M(L)$ is basic in $M$ and if the formula is true for $M, b$ and $\mu_{M}$ in a certain subset of the set of all $M$-dominant coweights, then it is also true for $(G, b, \mu)$. Thus it is enough to consider superbasic elements $b$, that is elements for which no $\sigma$-conjugate is contained in a proper Levi subgroup of $G$. They show in 5.9 that it is enough to consider the case that $G=G L_{h}$ for some $h$ and that $b$ is basic with $m=v_{t}(\operatorname{det}(b))$ prime to $h$. In this paper we prove Theorem 1.1 for this remaining case.

The strategy of the proof is as follows: We associate to the elements of $X_{\mu}(b)$ discrete invariants which we call extended semi-modules. This induces a decomposition of each connected component of $X_{\mu}(b)$ into finitely many locally closed subschemes. Their dimensions can be written as a combinatorial expression which only depends on the extended semi-module. By estimating these expressions we obtain the desired dimension formula.

For minuscule $\mu$, and over $\mathbb{Q}_{p}$, the group $J\left(\mathbb{Q}_{p}\right)$ acts transitively on the set of irreducible components of $X_{\mu}(b)$. As an application of the proof we show that for nonminuscule $\mu$, the action of $J(F)$ on this set may have more than one orbit.

\section{Notation and conventions}

From now on we use the following notation: Let $G=G L_{h}$ and let $A$ be the diagonal torus. Let $B$ be the Borel subgroup of lower triangular matrices. For $\mu, \mu^{\prime} \in X_{*}(A)_{\mathbb{Q}}$ we say that $\mu \preceq \mu^{\prime}$ if $\mu^{\prime}-\mu$ is a non-negative linear combination of positive coroots. As we may identify $X_{*}(A)_{\mathbb{Q}}$ with $\mathbb{Q}^{h}$, this induces a partial ordering on the latter set. An element $\mu=\left(\mu_{1}, \ldots, \mu_{h}\right) \in$ $X_{*}(A) \cong \mathbb{Z}^{h}$ is dominant if $\mu_{1} \leqslant \cdots \leqslant \mu_{h}$.

Let $N=L^{h}$ and let $M_{0} \subset N$ be the lattice generated by the standard basis $e_{0}, \ldots, e_{h-1}$. Then $K=G L_{h}\left(\mathcal{O}_{L}\right)=\operatorname{Stab}\left(M_{0}\right)$ and $g \mapsto g M_{0}$ defines a bijection

$$
X_{\mu}(b)(\bar{k}) \cong\left\{M \subset N \text { lattice } \mid \operatorname{inv}(M, b \sigma(M))=t^{\mu}\right\} .
$$

We define the volume of $M=g M_{0} \in X_{\mu}(b)$ to be $v_{t}(\operatorname{det}(g))$.

We assume $b$ to be superbasic. The Newton point $\nu \in X_{*}(A)_{\mathbb{Q}} \cong \mathbb{Q}^{h}$ of $b$ is then of the form $\nu=\left(\frac{m}{h}, \ldots, \frac{m}{h}\right) \in \mathbb{Q}^{h}$ with $(m, h)=1$. For $i \in \mathbb{Z}$ define $e_{i}$ by $e_{i+h}=t e_{i}$. We choose $b$ to be

$4^{\mathrm{e}}$ SÉRIE - TOME $39-2006-\mathrm{N}^{\circ} 3$ 
the representative of its $\sigma$-conjugacy class that maps $e_{i}$ to $e_{i+m}$ for all $i$. For superbasic $b$, the condition that the affine Deligne-Lusztig variety is nonempty, namely $\nu \preceq \mu$, is equivalent to $\sum \mu_{i}=m$. From now on we assume this.

For each central $\alpha \in X_{*}(A)$ there is the trivial isomorphism

$$
X_{\mu}(b) \rightarrow X_{\mu+\alpha}\left(t^{\alpha} b\right)
$$

We may therefore assume that all $\mu_{i}$ are nonnegative. For the lattices in (2.1), this implies that $b \sigma(M) \subseteq M$.

In the following we will abbreviate the right-hand side of the dimension formula for $X_{\mu}(b)$ by $d(b, \mu)$.

The set of connected components of $X$ is isomorphic to $\mathbb{Z}$, an isomorphism is given by mapping $g \in G L_{h}(L)$ to $v_{t}(\operatorname{det}(g))$. Let $X_{\mu}(b)^{i}$ be the intersection of the affine Deligne-Lusztig variety with the $i$-th connected component of $X$. Let $\pi \in G L_{h}(L)$ with $\pi\left(e_{i}\right)=e_{i+1}$ for all $i \in \mathbb{Z}$. Then $\pi$ commutes with $b \sigma$, and defines isomorphisms $X_{\mu}(b)^{i} \rightarrow X_{\mu}(b)^{i+1}$ for all $i$. Thus it is enough to determine the dimension of $X_{\mu}(b)^{0}$.

For superbasic $b$, an element of $J(F)$ is determined by its value at $e_{0}$. More precisely, $J(F)$ is the multiplicative subgroup of a central simple algebra over $F$. Hence $\operatorname{def}_{G}(b)=h-1$. If $v_{t}(\operatorname{det}(g))=i$ for some $g \in J(F)$, then $g$ induces isomorphisms between $X_{\mu}(b)^{j}$ and $X_{\mu}(b)^{j+i}$ for all $j$. On $X_{\mu}(b)^{0}$, we have an action of $\left\{g \in J(F) \mid v_{t}(\operatorname{det}(g))=0\right\}=J(F) \cap \operatorname{Stab}\left(M_{0}\right)$.

Remark 2.1. - To a vector $\psi=\left(\psi_{i}\right) \in \mathbb{Q}^{h}$ we associate the polygon in $\mathbb{R}^{2}$ that is the graph of the piecewise linear continuous function $f:[0, h] \rightarrow \mathbb{R}$ with $f(0)=0$ and slope $\psi_{i}$ on $[i-1, i]$. One can easily see that $d(b, \mu)$ is equal to the number of lattice points below the polygon corresponding to $\nu$ and (strictly) above the polygon corresponding to $\mu$.

\section{Extended semi-modules}

In this section we describe the combinatorial invariants which are used to decompose $X_{\mu}(b)^{0}$.

DEFINITION 3.1. - (1) Let $m$ and $h$ be coprime positive integers. A semi-module for $m$, $h$ is a subset $A \subset \mathbb{Z}$ that is bounded below and satisfies $m+A \subset A$ and $h+A \subset A$. Let $B=A \backslash(h+A)$. The semi-module is called normalized if $\sum_{a \in B} a=\frac{h(h-1)}{2}$.

(2) Let $\nu=\left(\frac{m}{h}, \ldots, \frac{m}{h}\right) \in \mathbb{Q}^{h}$. Let $\mu^{\prime}=\left(\mu_{1}^{\prime}, \ldots, \mu_{h}^{\prime}\right) \in \mathbb{N}^{h}$ not necessarily dominant with $\nu \preceq \mu^{\prime}$. A semi-module $A$ for $m, h$ is of type $\mu^{\prime}$ if the following condition holds: Let $b_{0}=$ $\min \{b \in B\}$ and let inductively $b_{i}=b_{i-1}+m-\mu_{i}^{\prime} h \in \mathbb{Z}$ for $i=1, \ldots, h$. Then $b_{0}=b_{h}$ and $\left\{b_{i} \mid i=0, \ldots, h-1\right\}=B$.

Remark 3.2. - Semi-modules are also used by de Jong and Oort in [2] to define a stratification of a moduli space of $p$-divisible groups whose rational Dieudonné modules are simple of slope $\frac{m}{h}$. In this case $\mu$ is minuscule, and they use semi-modules for $m, h-m$ to decompose the moduli space.

LEMMA 3.3. - If $A$ is a semi-module, then its translate $-\frac{\Sigma_{a \in B} a}{h}+\frac{h-1}{2}+A$ is the unique normalized translate of $A$. It is called the normalization of $A$. There is a bijection between the set of normalized semi-modules for $m$, $h$ and the set of possible types $\mu^{\prime} \in \mathbb{N}^{h}$ with $\nu \preceq \mu^{\prime}$.

Proof. - For the first assertion one only has to notice that the fact that the $h$ elements of $B$ are incongruent modulo $h$ implies that $\sum_{a \in B} a-\frac{h(h-1)}{2}$ is divisible by $h$. For the second assertion let $A$ be a normalized semi-module, let $b_{0}=\min \{a \in B\}$ and let inductively $b_{i}=b_{i-1}+m-\mu_{i}^{\prime} h$ where $\mu_{i}^{\prime}$ is maximal with $b_{i} \in A$. Then $b_{h}=b_{0}$ and $\left\{b_{i} \mid i=0, \ldots, h-1\right\}=B$. From 
$b_{0}<b_{i_{0}}$ for $i_{0}=1, \ldots, h-1$ we obtain $\sum_{i=1}^{i_{0}}\left(m-\mu_{i}^{\prime} h\right)>0$ for all $i_{0}<h$. Similarly, $b_{0}=b_{h}$ implies $\sum_{i=1}^{h} \mu_{i}^{\prime}=m$. This shows $\nu \preceq \mu^{\prime}$. As $m+A \subset A$, the $\mu_{i}^{\prime}$ are nonnegative. Given $\mu^{\prime}$ as above, the corresponding normalized semi-module $A$ can be constructed as follows: Let $b_{0}=0$, and inductively $b_{i}=b_{i-1}+m-\mu_{i}^{\prime} h$. Then $A$ is the normalization of $\left\{b_{i}+\alpha h \mid \alpha \in \mathbb{N}\right.$, $0 \leqslant i<h\}$.

Definition 3.4. - Let $m$ and $h$ be as before and let $\mu=\left(\mu_{i}\right) \in \mathbb{N}^{h}$ be dominant with $\sum \mu_{i}=m$. An extended semi-module $(A, \varphi)$ for $\mu$ is a normalized semi-module $A$ for $m$, $h$ together with a function $\varphi: \mathbb{Z} \rightarrow \mathbb{N} \cup\{-\infty\}$ with the following properties:

(1) $\varphi(a)=-\infty$ if and only if $a \notin A$.

(2) $\varphi(a+h) \geqslant \varphi(a)+1$ for all $a$.

(3) $\varphi(a) \leqslant \max \{n \mid a+m-n h \in A\}$ for all $a \in A$. If $b \in A$ for all $b \geqslant a$, then the two sides are equal.

(4) There is a decomposition of $A$ into a disjoint union of sequences $a_{j}^{1}, \ldots, a_{j}^{h}$ with $j \in \mathbb{N}$ and the following properties:

(a) $\varphi\left(a_{j+1}^{l}\right)=\varphi\left(a_{j}^{l}\right)+1$.

(b) If $\varphi\left(a_{j}^{l}+h\right)=\varphi\left(a_{j}^{l}\right)+1$, then $a_{j+1}^{l}=a_{j}^{l}+h$. Otherwise $a_{j+1}^{l}>a_{j}^{l}+h$.

(c) The $h$-tuple $\left(\varphi\left(a_{0}^{l}\right)\right)$ is a permutation of $\mu$.

An extended semi-module such that equality holds in (3) for all $a \in A$ is called cyclic.

Let $A$ be a normalized semi-module for $m, h$ and let $\mu^{\prime}$ be its type. Let $\mu=\mu_{\text {dom. }}^{\prime}$. Let $\varphi$ be such that (1) holds and that we have equality in (3) for all $a \in A$. Then in (2) the two sides are also equal for all $a \in A$. A decomposition of $A$ as in (4) is given by putting all elements into one sequence that are congruent modulo $h$. Hence $(A, \varphi)$ is a cyclic extended semi-module for $\mu$, called the cyclic extended semi-module associated to $A$.

Example 3.5. - We give an explicit example of a noncyclic extended semi-module for $m=4$, $h=5$, and $\mu=(0,0,0,2,2)$. Let $A$ be the normalized semi-module of type $(0,0,1,2,1)$. Then $B=A \backslash(5+A)$ consists of $-2,-1,2,5$, and 6 . Let $\varphi(-1)=0$ and $\varphi(a)=\max \{n \mid$ $a+m-n h \in A\}$ if $a \in A \backslash\{-1\}$. See also Fig. 1 that shows elements of $A$ marked by crosses and the corresponding values of $\varphi$. A decomposition of $A$ is given as follows: Three sequences are given by the elements of $A$ congruent to $-2,2$, and 5 modulo 5 , respectively. The forth sequence is given by all elements congruent to 4 modulo 5 and greater than -1 . The last sequence consists of the remaining elements -1 and $6,11,16, \ldots$

LEMMA 3.6. - If $(A, \varphi)$ is an extended semi-module for $\mu$, and if $\mu^{0}$ is the type of $A$, then $\mu_{\mathrm{dom}}^{0} \preceq \mu$. If $\mu_{\mathrm{dom}}^{0}=\mu$, then $(A, \varphi)$ is a cyclic extended semi-module.

Proof. - Let $\left(A, \varphi_{0}\right)$ be the cyclic extended semi-module associated to $A$. Let

$$
\left\{x_{1}, \ldots, x_{n}\right\}=\{a \in A \mid \varphi(a+h)>\varphi(a)+1\}
$$

\begin{tabular}{cccccccccccccccc}
\hline$a$ & & & -3 & -2 & -1 & 0 & 1 & 2 & 3 & 4 & 5 & 6 & & & \\
& $\ldots$ &. &. & $\times$ & $\times$ &. &. & $\times$ & $\times$ & $\times$ & $\times$ & $\times$ & $\times$ & $\times$ & $\cdots$ \\
$\varphi(a)$ & & $\cdots$ & $-\infty$ & 0 & 0 & $-\infty$ & $-\infty$ & 0 & 1 & 2 & 2 & 1 & 1 & 2 & $\cdots$ \\
\hline
\end{tabular}

Fig. 1. A noncyclic extended semi-module. 
with $x_{i}>x_{i+1}$ for all $i$. For $i \in\{1, \ldots, n\}$ let

$$
\varphi_{i}(a)= \begin{cases}-\infty & \text { if } a \notin A \\ \varphi(a) & \text { if } a \geqslant x_{i} \\ \varphi_{i}(a+h)-1 & \text { else }\end{cases}
$$

We show that $\left(A, \varphi_{i}\right)$ is an extended semi-module for some $\mu^{i}$ with $\mu_{\text {dom }}^{i-1} \preceq \mu_{\text {dom }}^{i}$ and $\mu_{\text {dom }}^{i-1} \neq \mu_{\text {dom }}^{i}$ for all $i \geqslant 1$. As $\varphi_{n}=\varphi$, it then follows that $\mu_{\text {dom }}^{0} \preceq \mu_{\text {dom }}^{n}=\mu$ with equality if and only if $n=0$, that is if $\varphi$ is cyclic.

The decomposition of $\left(A, \varphi_{i}\right)$ is defined as follows: For $a<x_{i}$, the successor of $a$ is $a+h$. Otherwise it is the successor from the decomposition of $(A, \varphi)$. From the properties of the decompositions for $\varphi_{0}$ and $\varphi$ one deduces that the decomposition satisfies the required properties. Let $n_{i} \geqslant 0$ be maximal with $x_{i}-n_{i} h \in A$ and let $\alpha_{i}=\varphi\left(x_{i}+h\right)-1-\varphi\left(x_{i}\right)>0$. Thus $\varphi_{i}$ is obtained from $\varphi_{i-1}$ by subtracting $\alpha_{i}$ from the values at $x_{i}, x_{i}-h, \ldots, x_{i}-n_{i} h$. From $\mu^{i-1}$ we obtain $\mu^{i}$ by replacing the two entries $\varphi_{i-1}\left(x_{i}-n_{i} h\right)=\varphi_{i-1}\left(x_{i}\right)-n_{i}$ and $\varphi_{i-1}\left(x_{i}\right)-\alpha_{i}+1$ (which is the value of $\varphi$ of the successor of $x_{i}$ in the sequence corresponding to $\varphi_{i}$ ) by $\varphi_{i-1}\left(x_{i}\right)-\alpha_{i}-n_{i}$ and $\varphi_{i-1}\left(x_{i}\right)+1$. As

$$
\varphi_{i-1}\left(x_{i}\right)-n_{i}, \varphi_{i-1}\left(x_{i}\right)-\alpha_{i}+1 \in\left(\varphi_{i-1}\left(x_{i}\right)-\alpha_{i}-n_{i}, \varphi_{i-1}\left(x_{i}\right)+1\right),
$$

we have $\mu_{\text {dom }}^{i-1} \preceq \mu_{\text {dom }}^{i}$ and $\mu_{\text {dom }}^{i-1} \neq \mu_{\text {dom }}^{i}$.

COROLLARY 3.7. - If $\mu$ is minuscule, then all extended semi-modules for $\mu$ are cyclic.

Proof. - Let $(A, \varphi)$ be such an extended semi-module. Let $\mu^{\prime}$ be the type of $A$. Then $\mu_{\text {dom }}^{\prime} \preceq \mu$, thus $\mu_{\mathrm{dom}}^{\prime}=\mu$. Hence the assertion follows from the preceding lemma.

LEMMA 3.8. - There are only finitely many extended semi-modules $(A, \varphi)$ for each $\mu$.

Proof. - Let $\mu^{\prime}$ be the type of the semi-module $A$. As $\mu_{\text {dom }}^{\prime} \preceq \mu$, there are only finitely many possible types and corresponding normalized semi-modules. For fixed $A$, the third condition for extended semi-modules determines all but finitely many values of $\varphi$. For the remaining values we have $0 \leqslant \varphi(a) \leqslant \max \{n \mid a+m-n h \in A\}$. Thus for each $A$ there are only finitely many possible functions $\varphi$ such that $(A, \varphi)$ is an extended semi-module for $\mu$.

\section{The decomposition of the affine Deligne-Lusztig variety}

Let $M \in X_{\mu}(b)^{0}$ be a lattice in $N$. In this section we associate to $M$ an extended semimodule for $\mu$. This leads to a paving of $X_{\mu}(b)^{0}$ by finitely many locally closed subschemes. For minuscule $\mu$, this decomposition of the set of lattices is the same as the one constructed by de Jong and Oort in [2], compare also [10, Section 5.1].

Let $m$ and $h$ be as in Section 2. Let $v \in N$ and recall that $t e_{i}=e_{i+h}$. Then we can write $v=\sum_{i \in \mathbb{Z}} \alpha_{i} e_{i}$ with $\alpha_{i} \in \bar{k}$ and $\alpha_{i}=0$ for small $i$. Let

$$
\begin{aligned}
I: N \backslash\{0\} & \rightarrow \mathbb{Z}, \\
v & \mapsto \min \left\{i \mid \alpha_{i} \neq 0\right\} .
\end{aligned}
$$

For a lattice $M \in X_{\mu}(b)^{0}$ we consider the set

$$
A=A(M)=\{I(v) \mid v \in M \backslash\{0\}\} .
$$


Then $A(M)$ is bounded below and $h+A(M) \subset A(M)$. As $b \sigma(M) \subset M$, we have $m+A(M) \subset$ $A(M)$, thus $A(M)$ is a semi-module for $m, h$. We have

$$
\operatorname{vol}(M)=|\mathbb{N} \backslash(A \cap \mathbb{N})|-|A \backslash(\mathbb{N} \cap A)|=0 .
$$

This implies that $\sum_{a \in B} a=\sum_{i=0}^{h-1} i$, thus $A$ is normalized.

Let further

$$
\begin{aligned}
\varphi=\varphi(M): \mathbb{Z} & \rightarrow \mathbb{N} \cup\{-\infty\}, \\
a & \mapsto \begin{cases}\max \left\{n \mid \exists v \in M \text { with } I(v)=a, t^{-n} b \sigma(v) \in M\right\} & \text { if } a \in A(M), \\
-\infty & \text { else. }\end{cases}
\end{aligned}
$$

Note that by the definition of $A(M)$, the set on the right-hand side is nonempty. As $b \sigma(M) \subset M$, the values of $\varphi$ are indeed in $\mathbb{N} \cup\{-\infty\}$.

LEMmA 4.1. - Let $M \in X_{\mu}(b)^{0}$. Then $(A(M), \varphi(M))$ is an extended semi-module for $\mu$.

Proof. - We already saw that $A(M)$ is a normalized semi-module. We have to check the conditions on $\varphi$. The first condition holds by definition. Let $v \in M$ with $I(v)=a$ be realizing the maximum for $\varphi(a)$. Then $t v \in M$ with $I(t v)=a+h$ implies that $\varphi(a+h) \geqslant \varphi(a)+1$, which shows (2). Let $v \in M$ with $I(v)=a$ and $t^{-\varphi(a)} b \sigma(v) \in M$. Then $I\left(t^{-\varphi(a)} b \sigma(v)\right)=$ $a+m-\varphi(a) h \in A(M)$, whence the first part of (3). Let $b \in A$ for all $b \geqslant a$. Let $n_{0}=\max \{n \mid$ $a+m-n h \in A\}$. Let $v^{\prime} \in M$ with $I\left(v^{\prime}\right)=a+m-n_{0} h$ and let $v=(b \sigma)^{-1}\left(t^{n_{0}} v^{\prime}\right) \in N$. Then $I(v)=a$, thus $v=\sum_{b \geqslant a} \alpha_{b} e_{b}$ for some $\alpha_{b} \in \bar{k}$. As $b \in A$ for all $b \geqslant a$, we also have $e_{b} \in M$ for all such $b$. Thus $v \in M$ with $t^{-n_{0}} b \sigma(v)=v^{\prime} \in M$. Hence $\varphi(a)=n_{0}$. It remains to show (4). For $a \in \mathbb{Z}$ and $\varphi_{0} \in \mathbb{N}$ let

$$
\widetilde{V}_{a, \varphi_{0}}=\left\{v \in M \mid v=0 \text { or } I(v) \geqslant a, t^{-\varphi_{0}} b \sigma(v) \in M\right\}
$$

and $V_{a, \varphi_{0}}=\widetilde{V}_{a, \varphi_{0}} / \widetilde{V}_{a, \varphi_{0}+1}$. Then $V_{a_{0}, \varphi_{0}}$ is a $\bar{k}$-vector space of dimension $\mid\left\{a \geqslant a_{0} \mid \varphi(a)=\right.$ $\left.\varphi_{0}\right\} \mid$. We construct the sequences by inductively sorting all elements $a \in A$ with $\varphi(a) \leqslant \varphi_{0}$ for some $\varphi_{0}$ : For $\varphi_{0}=\min \{\varphi(a) \mid a \in A\}$ we take each element $a$ with this value of $\varphi$ as the first element of a sequence. (At the end we will see that we did not construct more than $h$ sequences.) We now describe the induction step from $\varphi_{0}$ to $\varphi_{0}+1$ : If $v_{1}, \ldots, v_{i}$ is a basis of $V_{a, \varphi_{0}}$ for some $a$, then the $t v_{j}$ are linearly independent in $V_{a+h, \varphi_{0}+1}$. Thus $\operatorname{dim} V_{a, \varphi_{0}} \leqslant \operatorname{dim} V_{a+h, \varphi_{0}+1}$ for every $a$. Hence there are enough elements $a \in A$ with $\varphi(a)=\varphi_{0}+1$ to prolong all existing sequences such that conditions (a) and (b) are satisfied. We take the $a \in A$ with $\varphi(a)=\varphi_{0}+1$ that are not already in some sequence as first elements of new sequences. Inductively, this constructs sequences with properties (a) and (b). To show (c), let $a<b_{0}$. Then

$$
\begin{aligned}
\left|\left\{i \mid \mu_{i}=n\right\}\right| & =\operatorname{dim}_{\bar{k}} V_{a, n}-\operatorname{dim}_{\bar{k}} V_{a-h, n-1} \\
& =\left|\left\{a_{0}^{l} \mid \varphi\left(a_{0}^{l}\right)=n\right\}\right| .
\end{aligned}
$$

This also shows that we constructed exactly $h$ sequences.

For each extended semi-module $(A, \varphi)$ for $\mu$ let

$$
\mathcal{S}_{A, \varphi}=\{M \subset N \text { lattice } \mid A(M)=A, \varphi(M)=\varphi\} \subset X .
$$

LEMma 4.2. - The sets $\mathcal{S}_{A, \varphi}$ are contained in $X_{\mu}(b)^{0}$. They define a decomposition of $X_{\mu}(b)^{0}$ into finitely many disjoint locally closed subschemes. Especially, $\operatorname{dim} X_{\mu}(b)^{0}=$ $\max \left\{\operatorname{dim} \mathcal{S}_{A, \varphi}\right\}$.

$4^{e}$ SÉRIE - TOME $39-2006-\mathrm{N}^{\circ} 3$ 
Proof. - The last property in the definition of an extended semi-module shows that $(A, \varphi)$ determines $\mu$. Thus $\mathcal{S}_{A, \varphi} \subseteq X_{\mu}(b)^{0}$. Using Lemmas 3.8 and 4.1 it only remains to show that the subschemes are locally closed. The condition that $a \in A(M)$ is equivalent to $\operatorname{dim}(M \cap$ $\left.\left\langle e_{a}, e_{a+1}, \ldots\right\rangle\right) /\left(M \cap\left\langle e_{a+1}, e_{a+2}, \ldots\right\rangle\right)=1$. This is clearly locally closed. If $a$ is sufficiently large, it is contained in all extended semi-modules for $\mu$ and if $a$ is sufficiently small, it is not contained in any extended semi-module for $\mu$. Thus fixing $A$ is an intersection of finitely many locally closed conditions on $X_{\mu}(b)^{0}$, hence locally closed. Similarly, it is enough to show that $\varphi(a)<n$ for some $a \in A$ and $n \in \mathbb{N}$ is an open condition on $\{M \in X \mid b \sigma(M) \subset M$, $A(M)=A\} \subset X$. But this condition is equivalent to

$$
\left(\left\langle e_{i} \mid i \geqslant a\right\rangle \cap M \cap t^{n}(b \sigma)^{-1}(M)\right) /\left\langle e_{i} \mid i \geqslant a+1\right\rangle=(0),
$$

which is an open condition.

Let $(A, \varphi)$ be an extended semi-module for $\mu$. Let

$$
\mathcal{V}(A, \varphi)=\{(a, b) \in A \times A \mid b>a, \varphi(a)>\varphi(b)>\varphi(a-h)\} .
$$

THEOREM 4.3.-

(1) Let $A$ and $\varphi$ be as above. There exists a nonempty open subscheme $U(A, \varphi) \subseteq \mathbb{A}^{\mathcal{V}(A, \varphi)}$ and a morphism $U(A, \varphi) \rightarrow \mathcal{S}_{A, \varphi}$ that induces a bijection between the set of $\bar{k}$-valued points of $U(A, \varphi)$ and $\mathcal{S}_{A, \varphi}$. Especially, $\operatorname{dim}\left(\mathcal{S}_{A, \varphi}\right)=|\mathcal{V}(A, \varphi)|$.

(2) If $(A, \varphi)$ is a cyclic extended semi-module, then $U(A, \varphi)=\mathbb{A}^{\mathcal{V}(A, \varphi)}$.

Proof. - We denote the coordinates of a point $x$ of $\mathbb{A}^{\mathcal{V}(A, \varphi)}$ by $x_{a, b}$ with $(a, b) \in \mathcal{V}(A, \varphi)$. To define a morphism $\mathbb{A}^{\mathcal{V}(A, \varphi)} \rightarrow X$, we describe the image $M(x)$ of a point $x \in \mathbb{A}^{\mathcal{V}(A, \varphi)}(R)$ where $R$ is a $\bar{k}$-algebra. For each $a \in A$ we define an element $v(a) \in N_{R}=N \otimes_{\bar{k}} R$ of the form $v(a)=\sum_{b \geqslant a} \alpha_{b} e_{b}$ with $\alpha_{a}=1$. The $R \llbracket t \rrbracket$-module $M(x) \subset N_{R}$ will then be generated by the $v(a)$. We want the $v(a)$ to satisfy the following relations: For $a \in h+A$ we want

$$
v(a)=t v(a-h)+\sum_{(a, b) \in \mathcal{V}(A, \varphi)} x_{a, b} v(b) .
$$

Let $y=\max \{b \in B\}$. If $a=y$ we want

$$
v(a)=e_{a}+\sum_{(a, b) \in \mathcal{V}(A, \varphi)} x_{a, b} v(b) .
$$

For all other elements $a \in B$, we want the following equation to hold: Let $a^{\prime} \in A$ be minimal with $a^{\prime}+m-\varphi\left(a^{\prime}\right) h=a$. Then $v^{\prime}=t^{-\varphi\left(a^{\prime}\right)} b \sigma\left(v\left(a^{\prime}\right)\right) \in N_{R}$ with $I\left(v^{\prime}\right)=a$. Let

$$
v(a)=v^{\prime}+\sum_{(a, b) \in \mathcal{V}(A, \varphi)} x_{a, b} v(b) .
$$

Claim 1. - For every $x \in \mathbb{A}^{\mathcal{V}(A, \varphi)}(R)$ there are uniquely determined $v(a) \in N_{R}$ for all $a \in A$ satisfying (4.2) to (4.4).

We set

$$
v(a)=\sum_{j \in \mathbb{N}} \alpha_{a, j} e_{a+j}
$$


with $\alpha_{a, j} \in R$ and $\alpha_{a, 0}=1$ for all $a$. We solve the equations by induction on $j$. Assume that the $\alpha_{a, j}$ are determined for $j \leqslant j_{0}$ and such that the equations for $v(a)$ hold up to summands of the form $\beta_{j} e_{j}$ with $j>a+j_{0}$. To determine the $\alpha_{a, j_{0}+1}$, we write $a \equiv y+i m(\bmod h)$ and proceed by induction on $i \in\{0, \ldots, h-1\}$. For $i=0$ and $a=y$, the coefficient $\alpha_{a, j_{0}+1}$ is the uniquely determined element such that (4.3) holds up to summands of the form $\beta_{j} e_{j}$ with $j>j_{0}+1$. Note that by induction on $j$ and as $b>a$, the coefficient of $e_{y+j_{0}+1}$ on the right-hand side of the equation is determined. For $a=y+n h$ with $n>0$, the coefficients are similarly defined by (4.2). For $i>0$ and $a \in A$ minimal in this congruence class, the coefficient is determined by (4.4). Here, the coefficient of $e_{a+j_{0}+1}$ on the right-hand side of each equation is determined by induction on $i$ and $j$. For larger $a$ in this congruence class we use again (4.2). By passing to the limit on $j$, we obtain the uniquely defined $v(a) \in N_{R}$ solving the equations.

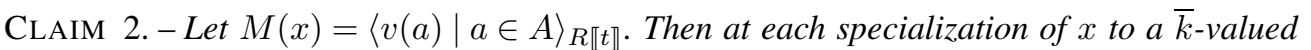
point $y$ we have $A=A(M(y))$ and $\varphi(M(y))(a) \geqslant \varphi(a)$ for all $a$.

From the definition of $M$ we immediately obtain $A \subseteq A(M(y))$. To show equality consider an element $v=\sum_{a} \alpha_{a} v(a) \in M(y)=M$. Write $v=\sum_{i \in \mathbb{Z}} b_{i} e_{i}$ with $b_{i} \in \bar{k}$. Let $i_{0}=\min \left\{I\left(\alpha_{a} v(a)\right)\right\}$. If $b_{i_{0}} \neq 0$, then $I(v)=i_{0} \in A$. Otherwise we consider $\sum_{\left\{a \mid I\left(\alpha_{a} v(a)\right)=i_{0}\right\}} \alpha_{a} v(a)$. Note that $I(v(a)) \equiv i_{0}(\bmod h)$ for all $a$ occurring in the sum. Then (4.2) shows that this sum can be written as a sum of $v(b)$ with $b>i_{0}$. Thus we may replace $i_{0}$ by a larger number. As $i \in A$ for all sufficiently large $i$, this shows that $I(v) \in A$, so $A(M)=A$.

Let $x \in \mathbb{A}^{\mathcal{V}(A, \varphi)}(\bar{k})$ and let $M=M(x)$. We show that $t^{-\varphi(a)} b \sigma(v(a)) \in M$ for all $a$. This means that $\varphi(M)(a) \geqslant \varphi(a)$ for all $a$. Consider the elements $a^{\prime} \in A$ that are minimal with $a^{\prime}+m-\varphi\left(a^{\prime}\right) h=a$ for some $a \in B \backslash\{y\}$. For these elements, the assertion follows from (4.4). If $a$ is minimal with $a+m-\varphi(a) h=y$, then $I\left(t^{-\varphi(a)} b \sigma(v(a))\right)=y$. As all $e_{i}$ with $i \geqslant y$ are in $M$, this element is also contained in $M$. If $\varphi(a)=\varphi(a-h)+1$ then $v(a)=t v(a-h)$ and the assertion holds for $a-h$ if and only if it holds for $h$. From this, we obtain the claim for all $a \in A$ with $\varphi(a)=\max \{n \mid a+m-n h \in A\}$. Especially, it follows for all sufficiently large elements of $A$. It remains to prove the claim for the finitely many elements $a \in A$ with $\max \{n \mid a+m-n h \in A\}>\varphi(a)$. We use decreasing induction on $a$ : Let $a$ be in this set, and assume that we know the assertion for all $a^{\prime}>a$. From (4.2) we obtain that

$$
\begin{aligned}
t^{-\varphi(a)} b \sigma(v(a)) & =t^{-\varphi(a)-1} b \sigma(t v(a)) \\
& =t^{-\varphi(a)-1} b \sigma\left(v(a+h)-\sum_{b>a+h, \varphi(a+h)>\varphi(b) \geqslant \varphi(a)+1} x_{a+h, b} v(b)\right) .
\end{aligned}
$$

By induction, the right-hand side is in $M$ and Claim 2 is shown.

As all $\mu_{i}$ are nonnegative, we constructed a morphism from $\mathbb{A}^{\mathcal{V}(A, \varphi)}$ to the subscheme $X_{A}$ of $X$ defined by $X_{A}(\bar{k})=\{M \mid A(M)=A, b \sigma(M) \subseteq M\}$.

CLAIM 3. - There is a nonempty open subscheme $U(A, \varphi)$ of $\mathbb{A} \mathcal{V}(A, \varphi)$ that is mapped to $\mathcal{S}_{A, \varphi}$. If $(A, \varphi)$ is cyclic, then $U(A, \varphi)=\mathbb{A}^{\mathcal{V}(A, \varphi)}$.

In general we do not have $\varphi(M)(a)=\varphi(a)$ for all $a$. The proof of Lemma 4.2 shows that $\varphi(M)(a) \leqslant \varphi(a)$ is an open condition on $X_{A}$, and thus on $\mathbb{A}^{\mathcal{V}(A, \varphi)}$. Let $U(A, \varphi)$ be the corresponding open subscheme, which is then mapped to $\mathcal{S}_{A, \varphi}$. We have to show that it is nonempty, thus to construct a point in $\mathbb{A}^{\mathcal{V}(A, \varphi)}$ where the corresponding function $\varphi(M)$ is equal to $\varphi$. If $\varphi(a)=\max \{n \mid a+m-n h \in A\}$, then $\varphi(M)(a)=\varphi(a)$. Especially, the two functions are equal for all $a$ if $(A, \varphi)$ is cyclic. In this case $U(A, \varphi)=\mathbb{A}^{\mathcal{V}(A, \varphi)}$. If $\varphi(a)+1=\varphi(a+h)$ and if $\varphi(M)(a+h)=\varphi(a+h)$, then $\varphi(M)(a+h)-1 \geqslant \varphi(M)(a) \geqslant \varphi(a)$ implies that $\varphi(M)(a)=\varphi(a)$. Thus it is enough to find a point where $\varphi(M)(a)=\varphi(a)$ for all $a \in A$ with 
$\varphi(a+h)>\varphi(a)+1$. For each such $a$ let $b_{a}$ be the successor in a decomposition of $(A, \varphi)$ into sequences. Then $\left(a+h, b_{a}\right) \in \mathcal{V}(A, \varphi)$. Let $x_{a+h, b_{a}}=1$ for these pairs and choose all other coefficients to be 0 . Then for this point and $a$ as before we have that $\varphi(M)(a)=\varphi\left(b_{a}\right)-1=$ $\varphi(a)$. Thus $U(A, \varphi)$ is nonempty.

CLAIM 4. - The map $U(A, \varphi) \rightarrow \mathcal{S}_{A, \varphi}$ defines a bijection on $\bar{k}$-valued points.

More precisely, we have to show that for each $M \in \mathcal{S}_{A, \varphi}$ there is exactly one $x \in U(A, \varphi)(\bar{k})$ such that $M$ contains a set of elements $v(a)$ for $a \in A$ with $I(v(a))=a$ and satisfying (4.2) to (4.4) for this $x$. The argument is similar as the construction of $v(a)$ for given $x$ : By induction on $j$ we will show the following assertion: There exist $x^{j}=\left(x_{a, b}^{j}\right) \in U(A, \varphi)(\bar{k})$ and $v_{j}(a) \in M$ for all $a$ with $t^{-\varphi(a)} b \sigma\left(v_{j}(a)\right) \in M$ and which satisfy Eqs. (4.2) to (4.4) for $x^{j}$ up to summands of the form $\beta_{n} e_{n}$ with $n>a+j$. Furthermore the $x_{a, b}^{j}$ with $b-a \leqslant j$ and the coefficients of $e_{n}$ in $v_{j}(a)$ for $n \leqslant a+j$ will be chosen independently of $j$ and only depending on $M$.

For $j=0$ choose any $x^{0} \in U(A, \varphi)(\bar{k})$ and $v_{0}(a) \in M$ with $I\left(v_{0}(a)\right)=a$, first coefficient 1 and $t^{-\varphi(a)} b \sigma\left(v_{0}(a)\right) \in M$. The existence of these $v_{0}(a)$ follows from $M \in X_{\mu}(b)$. Assume that the assertion is true for some $j_{0}$. For $n \leqslant j_{0}$ let $x_{a, a+n}^{j_{0}+1}=x_{a, a+n}^{j_{0}}$. We proceed again by induction on $i$ to define the coefficients for $a \equiv y+i m(\bmod h)$. Let $a=y$. Choose the coefficients $x_{y, y+n}^{j_{0}+1}$ with $n>j_{0}$ such that

$$
v_{j_{0}+1}(y)=e_{y}+\sum_{(y, y+n) \in \mathcal{V}(A, \varphi)} x_{y, y+n}^{j_{0}+1} v_{j_{0}}(y+n)
$$

satisfies $t^{-\varphi(y)} b \sigma\left(v_{j_{0}+1}(y)\right) \in M$. The definition of $\varphi=\varphi(M)$ shows that such coefficients exist and from $\varphi(y+n)<\varphi(y)$ it follows that they are unique. For the other elements $v(a)$ we proceed similarly: For those with $a-h \notin A$ we use Eq. (4.4), on the right-hand side with the values from the induction hypothesis, to define the new $v_{j_{0}+1}(a)$. For $a \in h+A$ we use (4.2). As we know that $t^{-\varphi(a-h)-1} b \sigma\left(t v_{j_{0}}(a-h)\right) \in M$, it is sufficient to consider the $b>a$ with $\varphi(a-h)<\varphi(b)<\varphi(a)$. At each step the coefficient of $e_{a+j_{0}+1}$ of the right-hand side is already defined by the induction hypothesis. It only depends on the $x_{a, a+n}^{j_{0}}$ and the coefficients of $e_{b+n}$ of $v_{j_{0}}(b)$ with $n \leqslant j_{0}$, hence only on $M$. The coefficients of $x^{j_{0}+1}$ are given by requiring that $t^{-\varphi(a)} b \sigma\left(v_{j_{0}+1}(a)\right) \in M$.

\section{Combinatorics}

In this section we estimate $|\mathcal{V}(A, \varphi)|$ to determine the dimension of the affine Deligne-Lusztig variety $X_{\mu}(b)$.

Remark 5.1. - For cyclic extended semi-modules we have $\varphi(a+h)=\varphi(a)+1$ for all $a \in A$. Thus

$$
\mathcal{V}(A, \varphi)=\left\{\left(b_{i}, b\right) \mid b_{i} \in B, b \in A, b>b_{i}, \varphi(b)<\varphi\left(b_{i}\right)\right\}
$$

where $B=A \backslash(h+A)$.

Proposition 5.2. - Let $(A, \varphi)$ be the cyclic extended semi-module associated to the normalized semi-module $A$ of type $\mu$. Then $|\mathcal{V}(A, \varphi)|=d(b, \mu)$.

Proof. - Recall that by $b_{0}$ we denote the minimal element of $A$ or $B$. Let $b_{i}$ be as in the definition of the type of $A$ and let $b_{h}=b_{0}$. First we show that 


$$
\begin{aligned}
\mathcal{V}(A, \varphi) & \rightarrow \mathbb{Z}, \\
\left(b_{i}, b\right) & \mapsto b-b_{i}+b_{h}
\end{aligned}
$$

induces a bijection between $\mathcal{V}(A, \varphi)$ and $\left\{a \notin A \mid a>b_{h}\right\}$. Let $b \in A$ for some $b>b_{i}$. Then $b-b_{i}+b_{i+1} \notin A$ if and only if $\left(b_{i}, b\right) \in \mathcal{V}(A, \varphi)$. Let $b_{i_{0}}=\max \left\{b_{i} \in B\right\}$. We have $b \in A$ for all $b \geqslant b_{i_{0}}$. Thus for every $b>b_{h}$ with $b \notin A$, there is an element $\left(b_{i}, b-b_{h}+b_{i}\right) \in \mathcal{V}(A, \varphi)$ for some $h>i \geqslant i_{0}$. Hence $\left\{a \notin A \mid a>b_{h}\right\}$ is in the image of the map. To show that it is injective and that its image is contained in $\left\{a \notin A \mid a>b_{h}\right\}$, it is enough to show that $\left(b_{i}, b\right) \in \mathcal{V}(A, \varphi)$ implies that $b-b_{i}+b_{j} \notin A$ for all $j \in\{i+1, \ldots, h\}$. Indeed, this ensures that $\left(b_{j}, b-b_{i}+b_{j}\right) \notin \mathcal{V}(A, \varphi)$ for all such $j$ and that $b-b_{i}+b_{h} \notin A$. We write $b=b_{l}+\alpha h$ for some $l$ and $\alpha$. Recall that $\varphi\left(b_{i}\right)=\mu_{i+1}$. As $\left(b_{i}, b_{l}+\alpha h\right) \in \mathcal{V}(A, \varphi)$, we have $\mu_{l+1}+\alpha<\mu_{i+1}$. Especially, $l<i$. This implies $\mu_{l+1}+\cdots+\mu_{l+\beta}+\alpha<\mu_{i+1}+\cdots+\mu_{i+\beta}$ for all $\beta \leqslant h-i$. Using the recurrence for the $b_{j}$, one sees that this implies $b-b_{i}+b_{i+\beta} \notin A$ for all $\beta \leqslant h-i$.

It remains to count the elements of $\left\{a \notin A \mid a>b_{0}\right\}$. As $h+A \subseteq A$, we have

$$
\left|\left\{a \notin A \mid a>b_{0}\right\}\right|=\left(\sum_{i=0}^{h-1} b_{i}-b_{0}-i\right) \cdot \frac{1}{h} .
$$

From the construction of $A$ from its type we obtain

$$
\begin{aligned}
& =\left(\sum_{i=0}^{h-1} \sum_{j=1}^{i}\left(m-\mu_{j} h\right)-i\right) \cdot \frac{1}{h} \\
& =\left(\sum_{i=0}^{h-1} \sum_{j=1}^{i} \frac{m}{h}-\mu_{j}\right)-\frac{h-1}{2} \\
& =d(b, \mu) .
\end{aligned}
$$

THEOREM 5.3. - Let $(A, \varphi)$ be an extended semi-module for $\mu$. Then $|\mathcal{V}(A, \varphi)| \leqslant d(b, \mu)$.

Proof of Theorem 5.3 for cyclic extended semi-modules. - We write $B=\left\{b_{0}, \ldots, b_{h-1}\right\}$ as in the definition of the type $\mu^{\prime}$ of $A$. As the extended semi-module is assumed to be cyclic, $\mu^{\prime}$ is a permutation of $\mu$. Using Remark 5.1 we see

$$
\begin{aligned}
|\mathcal{V}(A, \varphi)|= & \left|\left\{\left(b_{i}, a\right) \in B \times A \mid a>b_{i}, \varphi(a)<\varphi\left(b_{i}\right)\right\}\right| \\
= & \sum_{\left\{\left(b_{i}, b_{j}\right) \in B \times B \mid b_{j}>b_{i}, \mu_{j+1}^{\prime}<\mu_{i+1}^{\prime}\right\}} \mu_{i+1}^{\prime}-\mu_{j+1}^{\prime} \\
& +\left|\left\{\left(b_{i}, b_{j}+\alpha h\right) \mid b_{j}<b_{i}<b_{j}+\alpha h, \mu_{i+1}^{\prime}>\mu_{j+1}^{\prime}+\alpha\right\}\right| .
\end{aligned}
$$

We refer to these two summands as $S_{1}$ and $S_{2}$.

Let $\left(\tilde{b}_{0}, \tilde{\mu}_{1}\right), \ldots,\left(\tilde{b}_{h-1}, \tilde{\mu}_{h}\right)$ be the set of pairs $\left(b_{0}, \mu_{1}^{\prime}\right), \ldots,\left(b_{h-1}, \mu_{h}^{\prime}\right)$, but ordered by the size of $b_{i}$. That is, $\tilde{b}_{i}<\tilde{b}_{i+1}$ for all $i$. Let

$$
\begin{aligned}
f: B & \rightarrow B, \\
b_{i} & \mapsto b_{i+1}=b_{i}+m-\mu_{i+1}^{\prime} h
\end{aligned}
$$

where we identify $b_{h}$ with $b_{0}$. This defines a permutation of $B$. From the ordering of the $\tilde{b}_{i}$ we obtain $\sum_{i=0}^{i_{0}} f\left(\tilde{b}_{i}\right) \geqslant \sum_{i=0}^{i_{0}} \tilde{b}_{i}$ for all $i_{0}$. As $f\left(\tilde{b}_{i}\right)=\tilde{b}_{i}+m-\tilde{\mu}_{i+1} h$, this is equivalent to $\sum_{i=1}^{i_{0}+1} \tilde{\mu}_{i} \leqslant\left(i_{0}+1\right) \frac{m}{h}$ for all $i_{0}$. We thus have $\nu \preceq \tilde{\mu} \preceq \mu$. 
Recall the interpretation of $d(b, \mu)$ from Remark 2.1. We show that $S_{1}$ is equal to the number of lattice points above $\mu$ and on or below $\tilde{\mu}$. The second summand $S_{2}$ will be less or equal to the number of lattice points above $\tilde{\mu}$ and below $\nu$. Then the theorem follows for cyclic extended semi-modules.

We have $S_{1}=\sum_{i<j} \max \left\{\tilde{\mu}_{i+1}-\tilde{\mu}_{j+1}, 0\right\}$. Consider this sum for any permutation $\tilde{\mu}$ of $\mu$. If we interchange two entries $\tilde{\mu}_{i}$ and $\tilde{\mu}_{i+1}$ with $\tilde{\mu}_{i}>\tilde{\mu}_{i+1}$, the sum is lessened by the difference of these two values. There are also exactly $\tilde{\mu}_{i}-\tilde{\mu}_{i+1}$ lattice points on or below $\tilde{\mu}$ and above the polygon corresponding to the permuted vector. If $\tilde{\mu}=\mu$, both $S_{1}$ and the number of lattice points above $\mu$ and on or below $\tilde{\mu}$ are 0 . Thus by induction $S_{1}$ is equal to the claimed number of lattice points.

The last step is to estimate $S_{2}$. It is enough to construct a decreasing sequence (with respect to $\preceq$ ) of $\psi^{i} \in \mathbb{Q}^{h}$ for $i=0, \ldots, h-1$ with $\psi^{0}=\tilde{\mu}$ and $\psi^{h-1}=\nu$ such that the number of lattice points above $\psi^{i}$ and on or below $\psi^{i+1}$ is greater or equal to the number of pairs $\left(\tilde{b}_{i+1}, \tilde{b}_{j}+\alpha h\right)$ contributing to $S_{2}$. Note that the $\psi^{i}$ will no longer be lattice polygons. Let $f_{i}: B \rightarrow B$ be defined as follows: For $j>i$ let $f_{i}\left(\tilde{b}_{j}\right)=f\left(\tilde{b}_{j}\right)$. Let $\left\{f_{i}\left(\tilde{b}_{j}\right) \mid 0 \leqslant j \leqslant i\right\}$ be the set of $f\left(\tilde{b}_{j}\right)$, but sorted increasingly. Let $\psi^{i}=\left(\psi_{j}^{i}\right)$ be such that $f_{i}\left(\tilde{b}_{j}\right)=\tilde{b}_{j}+m-\psi_{j+1}^{i} h$, i.e.

$$
\psi_{j+1}^{i}=\frac{\tilde{b}_{j}+m-f_{i}\left(\tilde{b}_{j}\right)}{h}=\frac{m}{h}-\frac{f_{i}\left(\tilde{b}_{j}\right)-\tilde{b}_{j}}{h} .
$$

Similarly as for $\nu \preceq \tilde{\mu}$ one can show that

$$
\nu \preceq \psi^{i+1} \preceq \psi^{i} \preceq \tilde{\mu}
$$

for all $i$. As $f_{0}=f$ and $f_{h-1}=\mathrm{id}$, we have $\psi^{0}=\tilde{\mu}$ and $\psi^{h-1}=\nu$. It remains to count the lattice points between $\psi^{i}$ and $\psi^{i+1}$. To pass from $f_{i}$ to $f_{i+1}$ we have to interchange the value $f\left(\tilde{b}_{i+1}\right)$ with all larger $f_{i}\left(\tilde{b}_{j}\right)$ with $j \leqslant i$. Thus to pass from the polygon associated to $\psi^{i}$ to the polygon of $\psi^{i+1}$ we have to change the value at $j$ by $\left(f_{i}\left(\tilde{b}_{j}\right)-f\left(\tilde{b}_{i+1}\right)\right) / h$, and that for all $j \leqslant i$ with $f_{i}\left(\tilde{b}_{j}\right)>f\left(\tilde{b}_{i+1}\right)$. Thus there are at least

$$
\sum_{j \leqslant i, f_{i}\left(\tilde{b}_{j}\right)>f\left(\tilde{b}_{i+1}\right)}\left\lfloor\frac{f_{i}\left(\tilde{b}_{j}\right)-f\left(\tilde{b}_{i+1}\right)}{h}\right\rfloor=\sum_{j \leqslant i, f\left(\tilde{b}_{j}\right)>f\left(\tilde{b}_{i+1}\right)}\left\lfloor\frac{f\left(\tilde{b}_{j}\right)-f\left(\tilde{b}_{i+1}\right)}{h}\right\rfloor
$$

lattice points above $\psi^{i}$ and on or below $\psi^{i+1}$. For fixed $i$ and $j<i+1$, the set of pairs $\left(\tilde{b}_{i+1}, \tilde{b}_{j}+\alpha h\right)$ contributing to $S_{2}$ is in bijection with $\left\{\alpha \geqslant 1 \mid f\left(\tilde{b}_{j}\right)-\alpha h>f\left(\tilde{b}_{i+1}\right)\right\}$. The cardinality of this set is at most $\left\lfloor\frac{f\left(\tilde{b}_{j}\right)-f\left(\tilde{b}_{i+1}\right)}{h}\right\rfloor$ which proves that $S_{2}$ is not greater than the number of lattice points between $\tilde{\mu}$ and $\nu$.

Example 5.4. - We give an example of a cyclic semi-module $(A, \varphi)$ where the type of $A$ is not dominant but where $|\mathcal{V}(A, \varphi)|=d(b, \mu)$. Let $m=4, h=5$, and $\mu=(0,0,1,1,2)$. Let $(A, \varphi)$ be the cyclic extended semi-module associated to the normalized semi-module of type $(0,0,1,2,1)$. Note that $A$ is the same semi-module as in Example 3.5. Then the dimension of the corresponding subscheme is

$$
|\mathcal{V}(A, \varphi)|=|\{(-1,2),(5,6),(5,7)\}|=d(b, \mu) .
$$

Proof of Theorem 5.3. - Let $(A, \varphi)$ be an extended semi-module for $\mu$. Let $\varphi_{i}$ and $\mu^{i}$ be the sequences constructed in the proof of Lemma 3.6. By induction on $i$ we show that 
$\left|\mathcal{V}\left(A, \varphi_{i}\right)\right| \leqslant d\left(b, \mu^{i}\right)$. For $i=0$, the extended semi-module $\left(A, \varphi_{0}\right)$ is cyclic, hence the assertion is already shown.

We use the notation of the proof of Lemma 3.6. The description of the difference between $\mu^{i}$ and $\mu^{i-1}$ given there shows that

$$
\begin{aligned}
d\left(b, \mu^{i}\right)-d\left(b, \mu^{i-1}\right)= & \sum_{l=1}^{h} \sum_{j=1}^{l}\left(\mu_{\mathrm{dom}, j}^{i-1}-\mu_{\mathrm{dom}, j}^{i}\right) \\
= & \left(\left|\left\{\mu_{j}^{i-1} \in\left(\varphi_{i-1}\left(x_{i}\right)-\alpha_{i}-n_{i}, \varphi_{i-1}\left(x_{i}\right)+1\right)\right\}\right|-1\right) \\
& \times \min \left\{\alpha_{i}, n_{i}+1\right\} .
\end{aligned}
$$

We denote this difference by $\Delta$. To show that $\left|\mathcal{V}\left(A, \varphi_{i}\right)\right|-\left|\mathcal{V}\left(A, \varphi_{i-1}\right)\right| \leqslant \Delta$ we use the decomposition into sequences $a_{j}^{l}$ of the extended semi-module $\left(A, \varphi_{i-1}\right)$. Using the definition of $\mathcal{V}(A, \varphi)$ and the description of the difference between $\varphi_{i}$ and $\varphi_{i-1}$ from the proof of Lemma 3.6 one obtains

$$
\left|\mathcal{V}\left(a, \varphi_{i}\right)\right|-\left|\mathcal{V}\left(a, \varphi_{i-1}\right)\right|=S_{1}+S_{2}+S_{3}
$$

where

$$
\begin{aligned}
& S_{1}=\left|\left\{\left(x_{i}+h, b\right) \mid b \in A, b>x_{i}+h, \varphi_{i-1}\left(x_{i}\right)+1>\varphi_{i-1}(b)>\varphi_{i-1}\left(x_{i}\right)-\alpha_{i}\right\}\right|, \\
& S_{2}=\mid\left\{\left(b, x_{i}-\delta h\right) \mid b \in B \backslash\left\{x_{i}-n_{i} h\right\}, b<x_{i}-\delta h, \delta \in\left\{0, \ldots, n_{i}\right\}\right. \\
& \left.\qquad \varphi_{i-1}\left(x_{i}\right)-\delta-\alpha_{i}<\varphi_{i-1}(b) \leqslant \varphi_{i-1}\left(x_{i}\right)-\delta\right\} \mid, \\
& S_{3}=-\left|\left\{\left(x_{i}-n_{i} h, b\right) \mid b>x_{i}-n_{i} h, \varphi_{i-1}\left(x_{i}\right)-n_{i}>\varphi_{i-1}(b) \geqslant \varphi_{i-1}\left(x_{i}\right)-n_{i}-\alpha_{i}\right\}\right| .
\end{aligned}
$$

Here we used that $a \leqslant x_{i}$ implies that $\varphi_{i-1}(a+h)=\varphi_{i-1}(a)+1$. For each sequence $a_{j}^{l}$ of the extended semi-module $\left(A, \varphi_{i-1}\right)$ we use $S_{1, l}, S_{2, l}$, and $S_{3, l}$ for the contributions of pairs with $b \in\left\{a_{j}^{l}\right\}$ to the three summands. Furthermore we write $S^{l}=S_{1, l}+S_{2, l}+S_{3, l}$. We show the following assertions: If $\varphi_{i-1}\left(a_{0}^{l}\right) \notin\left(\varphi_{i-1}\left(x_{i}\right)-\alpha_{i}-n_{i}, \varphi_{i-1}\left(x_{i}\right)+1\right)$ or if $a_{0}^{l}=x_{i}-n_{i} h$, then $S^{l}=0$. Otherwise, $S^{l} \leqslant \min \left\{\alpha_{i}, n_{i}+1\right\}$. Then the theorem follows from property (4c) of extended semi-modules.

To determine the $S^{l}$, we consider the following cases:

Case 1. - $\varphi_{i-1}\left(a_{0}^{l}\right) \geqslant \varphi_{i-1}\left(x_{i}\right)+1$. In this case it is easy to see that $S_{1, l}=S_{2, l}=S_{3, l}=0$.

Case 2. - $a_{0}^{l}>x_{i}$. This implies that $S_{2, l}=0$. If $\varphi_{i-1}\left(a_{0}^{l}\right) \leqslant \varphi_{i-1}\left(x_{i}\right)-n_{i}-\alpha_{i}$, then $S_{1, l}+S_{3, l}=\alpha_{i}-\alpha_{i}=0$. Let now $\varphi_{i-1}\left(a_{0}^{l}\right) \in\left(\varphi_{i-1}\left(x_{i}\right)-\alpha_{i}-n_{i}, \varphi_{i-1}\left(x_{i}\right)+1\right)$. Then

$$
S_{1, l}+S_{3, l} \leqslant\left|\left\{a_{j}^{l} \mid \varphi_{i-1}\left(x_{i}\right)+1>\varphi_{i-1}\left(a_{j}^{l}\right) \geqslant \max \left\{\varphi_{i-1}\left(x_{i}\right)-\alpha_{i}+1, \varphi_{i-1}\left(x_{i}\right)-n_{i}\right\}\right\}\right| .
$$

As $\varphi_{i-1}\left(a_{j+1}^{l}\right)=\varphi_{i-1}\left(a_{j}^{l}\right)+1$ for all $j$, the right-hand side is less or equal to $\min \left\{\alpha_{i}, n_{i}+1\right\}$.

Case 3. $-\quad a_{0}^{l}=x_{i}-n_{i} h$. This sequence starts with $x_{i}-n_{i} h, \ldots, x_{i}, x_{i}+h$. (Recall that the sequences $\left\{a_{j}^{l}\right\}$ for $\varphi_{i-1}$ are of this easy form with stepwidth $h$ as long as $a_{j}^{l} \leqslant x_{i}<x_{i-1}$.) Note that within one sequence $a_{j}^{l}>a_{j^{\prime}}^{l}$ implies $\varphi_{i-1}\left(a_{j}^{l}\right)>\varphi_{i-1}\left(a_{j^{\prime}}^{l}\right)$. Hence this special sequence does not make any contribution, as in $S^{l}$ we only consider pairs where both elements are in the sequence starting with $x_{i}-n_{i} h$.

Case 4. - $a_{0}^{l}<x_{i}$, but not congruent to $x_{i}$ modulo $h$. Again $a_{j+1}^{l}=a_{j}^{l}+h$ if $a_{j}^{l} \leqslant x_{i}$. We first assume that $\varphi_{i-1}\left(a_{0}^{l}\right) \leqslant \varphi_{i-1}\left(x_{i}\right)-n_{i}-\alpha_{i}$. Then $S_{2, l}=0$. Assume that $b=a_{j}^{l}$ contributes 
to $S_{1, l}$. Then $j \geqslant n_{i}+1$ and $a_{j}^{l}>x_{i}+h$. If $a_{0}^{l}<x_{i}-n_{i} h$, then $\left[x_{i}-n_{i} h, x_{i}+h\right]$ contains $n_{i}+1$ elements of the sequence. Thus in all cases $a_{j-n_{i}-1}^{l}>x_{i}-n_{i} h$. This element then leads to a contribution to $S_{3, l}$, as $\varphi_{i-1}\left(a_{j-n_{i}-1}^{l}\right)=\varphi_{i-1}\left(a_{j}^{l}\right)-n_{i}-1$. In the other direction, if $a_{j}^{l}$ contributes to $S_{3, l}$, then $a_{j+n_{i}+1}^{l}$ contributes to $S_{1, l}$. Thus $S^{l}=0$. We now assume that $\varphi_{i-1}\left(a_{0}^{l}\right) \in\left(\varphi_{i-1}\left(x_{i}\right)-\alpha_{i}-n_{i}, \varphi_{i-1}\left(x_{i}\right)+1\right)$. Let $n$ be maximal with $a_{n}^{l}=a_{0}^{l}+n h<x_{i}$. Then we have

$$
\begin{aligned}
S_{1, l} & =\left|\left\{a_{j}^{l} \mid j>n+1, \varphi_{i-1}\left(x_{i}\right) \geqslant \varphi_{i-1}\left(a_{0}^{l}\right)+j>\varphi_{i-1}\left(x_{i}\right)-\alpha_{i}\right\}\right|, \\
S_{2, l} & =\left|\left\{a_{j}^{l} \mid 0 \leqslant j \leqslant \min \left\{n, n_{i}\right\}, \varphi_{i-1}\left(x_{i}\right) \geqslant \varphi_{i-1}\left(a_{0}^{l}\right)+j>\varphi_{i-1}\left(x_{i}\right)-\alpha_{i}\right\}\right|, \\
S_{3, l} & =-\left|\left\{a_{j}^{l} \mid j \geqslant \max \left\{n-n_{i}+1,0\right\}, \varphi_{i-1}\left(x_{i}\right)-n_{i}>\varphi_{i-1}\left(a_{0}^{l}\right)+j\right\}\right| \\
& =-\left|\left\{a_{j}^{l} \mid j>\max \left\{n+1, n_{i}\right\}, \varphi_{i-1}\left(x_{i}\right) \geqslant \varphi_{i-1}\left(a_{0}^{l}\right)+j\right\}\right| .
\end{aligned}
$$

Thus

$$
S^{l} \leqslant S_{1, l}+S_{2, l} \leqslant\left\{j \mid \varphi_{i-1}\left(x_{i}\right) \geqslant \varphi_{i-1}\left(a_{0}^{l}\right)+j>\varphi_{i-1}\left(x_{i}\right)-\alpha_{i}\right\}=\alpha_{i} .
$$

If $n+1 \geqslant n_{i}$, then $S_{1, l}+S_{3, l} \leqslant 0$. Thus $S^{l} \leqslant S_{2, l} \leqslant n_{i}+1$. If $n_{i}>n+1$ then $S_{1, l}+S_{3, l} \leqslant$ $n_{i}-n-1$ and $S_{2, l} \leqslant n+1$. Hence in both cases $S^{l} \leqslant \min \left\{\alpha_{i}, n_{i}+1\right\}$.

Example 5.5. - Example 3.5 describes a noncyclic extended semi-module $(A, \varphi)$ for $\mu=(0,0,0,2,2)$ such that

$$
|\mathcal{V}(A, \varphi)|=|\{(5,6),(5,7),(4,6),(4,7)\}|=d(b, \mu) .
$$

Proof of Theorem 1.1. - Lemma 4.2 and Theorem 4.3 imply that $\operatorname{dim} X_{\mu}(b)^{0}=\max |\mathcal{V}(A, \varphi)|$. In Proposition 5.2 we give a pair with $|\mathcal{V}(A, \varphi)|=d(b, \mu)$. Theorem 5.3 shows that the maximum is at most $d(b, \mu)$. Together we obtain $\operatorname{dim} X_{\mu}(b)=d(b, \mu)$.

\section{Irreducible components}

COROLLARY 6.1. - Let $G=G L_{h}$, let $b$ be superbasic and $\nu \preceq \mu$. Then the action of $J(F)$ on the set of irreducible components of $X_{\mu}(b)$ has only finitely many orbits.

Proof. - It is enough to consider the intersection of the orbits with the set of irreducible components of $X_{\mu}(b)^{0}$. Theorem 4.3 implies that each $\mathcal{S}_{A, \varphi}$ is irreducible. Thus the corollary follows from Lemma 3.8 .

Example 6.2. - We give two examples to show that even for superbasic $b$, the irreducible components of $X_{\mu}(b)$ are in general not permuted transitively by $J(F)$. The description of $J(F)$ in Section 2 implies that $A(g M)=A(M)$ and $\varphi(g M)=\varphi(M)$ for each $g \in J(F)$ with $v_{t}(\operatorname{det}(g))=0$. First we consider the example $m=4, h=5$, and $\mu=(0,0,1,1,2)$. It is enough to find two extended semi-modules for $\mu$ leading to subschemes of dimension $d(b, \mu)=3$. Indeed, the subschemes corresponding to different extended semi-modules are disjoint and lead to irreducible components in different $J(F)$-orbits. One such extended semi-module is the cyclic extended semi-module considered in Proposition 5.2. A second extended semi-module $(A, \varphi)$ is given in Example 5.4. Here, $A$ is of type $(0,0,1,2,1)$, hence different from the semi-module considered before.

For the second example let $m=4, h=5$, and $\mu=(0,0,0,2,2)$. Here the two extended semi-modules for $\mu$ leading to subschemes of dimension $d(b, \mu)=4$ are the ones considered 
in Proposition 5.2 and Examples 3.5 and 5.5. The corresponding semi-modules are different as they are of type $(0,0,0,2,2)$ and $(0,0,1,2,1)$.

\section{Acknowledgements}

I am grateful to M. Rapoport for his encouragement and helpful comments. I thank R. Kottwitz and Ngô B.-C. for their interest in my work. This work was completed during a stay at the Universite Paris-Sud at Orsay which was supported by a fellowship within the Postdoc-Program of the German Academic Exchange Service (DAAD). I want to thank the Université Paris-Sud for its hospitality.

\section{REFERENCES}

[1] Görtz U., Haines Th.J., Kottwitz R.E., Reuman D.C., Dimensions of some affine DeligneLusztig varieties, Ann. Scient. Éc. Norm. Sup. 39 (3) (2006), math.AG/0504443.

[2] De Jong A.J., Oort F., Purity of the stratification by Newton polygons, J. Amer. Math. Soc. 13 (2000) 209-241.

[3] Kottwitz R.E., Isocrystals with additional structure, Comp. Math. 56 (1985) 201-220.

[4] Kotтwitz R.E., Dimensions of Newton strata in the adjoint quotient of reductive groups, math.AG/0601196.

[5] Kottwitz R.E., Rapoport M., On the existence of F-crystals, Comment. Math. Helv. 78 (2003) 153-184.

[6] RAPOPORT M., A positivity property of the Satake isomorphism, Manuscripta Math. 101 (2) (2000) 153-166.

[7] RAPOPORT M., A guide to the reduction modulo $p$ of Shimura varieties, Astérisque 298 (2005) 271318.

[8] Reuman D.C., Determining whether certain affine Deligne-Lusztig sets are empty, PhD thesis, Chicago 2002, math.RT/0211434.

[9] REUMAN D.C., Formulas for the dimensions of some affine Deligne-Lusztig varieties, Michigan Math. J. 52 (2004) 435-451.

[10] Viehmann E., Moduli spaces of $p$-divisible groups, math.AG/0502320.

(Manuscrit reçu le 3 novembre 2005; accepté, après révision, le 6 avril 2006.)

Eva VIEHMANN

Mathematisches Institut der Universität Bonn,

Beringstrasse 1,

53115 Bonn, Germany

E-mail: viehmann@math.uni-bonn.de

$4^{\text {e }}$ SÉRIE - TOME $39-2006-\mathrm{N}^{\circ} 3$ 